\title{
BIOMETRIC IDENTITY VERIFICATION USING INTRA-BODY PROPAGATION SIGNAL
}

\author{
Isao Nakanishi, Yasuhiro Yorikane, Yoshio Itoh, Yutaka Fukui \\ Faculty of Engineering, Tottori University \\ 4-101 Koyama-minami, Tottori, 680-8552 Japan
}

\begin{abstract}
We propose to utilize an electromagnetic wave through a human body as biometrics. The electromagnetic wave (intrabody propagation signal) is generated at a relatively shallow depth in the human body through a pair of electrodes pasted on the human skin. The biological tissue of each individual human being is different from that of others, so that the transfer characteristic of the intra-body propagation signal is also different mutually. By using such a difference, it is expected to authenticate personal identification. In addition, liveness detection can be realized simultaneously using the intra-body propagation signal. It is effective on the detection of spoofing using artificial bodies. In this paper, we examine the individual feature in the intra-body propagation signal based on the spectrum analysis. As a result, the verification rate of $58 \%$ is obtained using the similarity of the power spectrum especially in the 30-60 MHz sub-band.
\end{abstract}

\section{INTRODUCTION}

Individual verification is to confirm that a user is genuine and to prevent spoofing attacks. As technologies to certify an individual, a password and a magnetic card have been used because of their easiness. However, they tend to be forgotten or lost. Furthermore, the counterfeit and the theft can happen. On the other hand, the biometrics authentication has attracted attention because the above-mentioned various problems can be solved or reduced by the biometrics [1].

There are the static biometrics such as a fingerprint and an iris, and the dynamic biometrics such as a voiceprint and a signature. Especially, the authentication system using the fingerprint is used in a lot of fields including the cellular phone. On the other hand, there has been reported that an authentication system using the fingerprint is circumvented by a counterfeit finger made by gum [2]. In order to exclude such an artifact, some additional sensor or function of liveness detection is required in the authentication system.

In this paper, we propose to utilize an electromagnetic wave through a human body as the biometrics. The electromagnetic wave is generated at a relatively shallow depth in a human body through a pair of electrodes pasted on the human skin. On the other hand, the propagated electromagnetic wave (intra-body propagation signal) is detected through another pair of electrodes which keeps a distance from the previous pair. The biological tissue of each individual human being is different from that of others, so that the transfer characteristic of the intra-body propagation signal is also different mutually. By using such a difference, it is expected to authenticate personal identification. In addition, using the intra-body propagation signal, liveness detection can be realized with no additional sensor.

This paper is organized as follows. In Sect. 2, we explain the method of making an intra-body propagation signal. Then, we examine the intra-body propagation signal in a frequency domain in Sect. 3. In Sect. 4, the evaluation based on similarity is examined and then the performance of authentication is evaluated in Sect. 5. Finally, Sect. 6 presents a conclusion.

\section{INTRA-BODY PROPAGATION SIGNAL}

\subsection{Transmission method}

Figure 1 shows three transmission methods used in an intrabody communication [3]. The simple circuit type treats a human body as a conducting wire and constitutes a closed circuit with an external circuit. This type is used for bioinstrumentation such as a body fat meter or an electrocardiogram. The electrostatic coupling type also regards a human body as a conducting wire but the human body is electrostatically coupled with the ground. The waveguide type regards a human body as a waveguide, where an input signal fed at input electrodes is propagated as an electromagnetic wave and it is extracted at output electrodes as an output signal. It is an advantage that the waveguide type is insensitive to environmental disturbances. In addition, wide frequency band signals can be transmitted in the waveguide type. Resultingly, we use the waveguide type in the following.

\subsection{Influence of electromagnetic waves on human health}

The influence of electromagnetic waves from electric equipments on a human being has been investigated but it is not still cleared. The frequency band used in this study is under the ultrahigh frequency band (UHF) and its wavelength is longer 


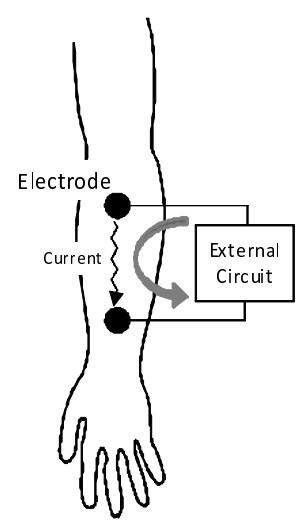

(a) Simple Circuit Type

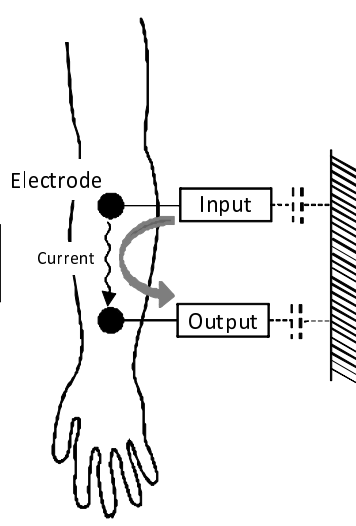

(b) Electrostatic Coupling Type

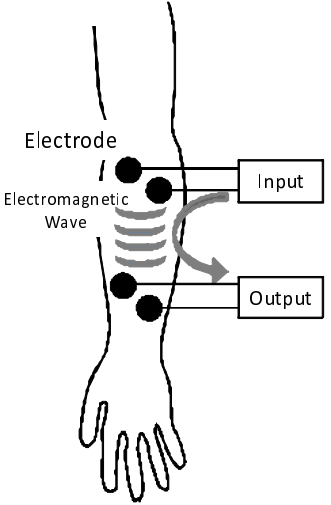

(c) Waveguide Type

Fig. 1. Transmission methods in intra-body communication.

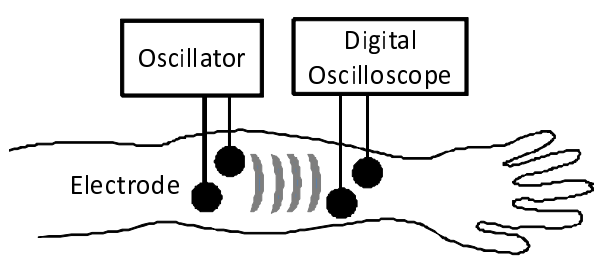

Fig. 2. Measurement system of intra-body propagation signals.

than that of gamma ray or X-rays; therefore, the electromagnetic wave has no danger to a human body.

\section{CHARACTERISTICS OF INTRA-BODY PROPAGATION SIGNALS}

In this section, we show the characteristics of intra-body propagation signals. Especially, the intra-class variation of one individual and the inter-class variation between individuals are examined on the spectrum analysis of the intra-body propagation signal. Moreover, the function of liveness detection is verified comparing the spectrum of a human body with those of other materials.

\subsection{Measuring system of intra-body propagation signals}

Figure 2 shows a measurement system of the intra-body propagation signal. A signal is fed from an oscillator to a pair of electrodes (a signal electrode and a GND one) pasted on a human body. On the other hand, a propagated electromagnetic wave (intra-body propagation signal) is detected through another pair of electrodes that keeps a distance from the previous pair. The detected signal is digitized by a digital oscilloscope and a FFT (Fast Fourier Transform) is performed for the spectrum analysis.

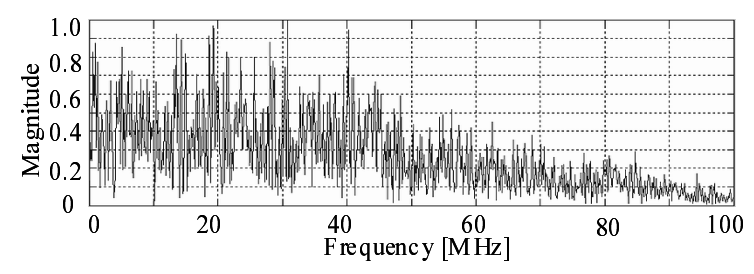

Fig. 3. Spectrum of a transmission signal.

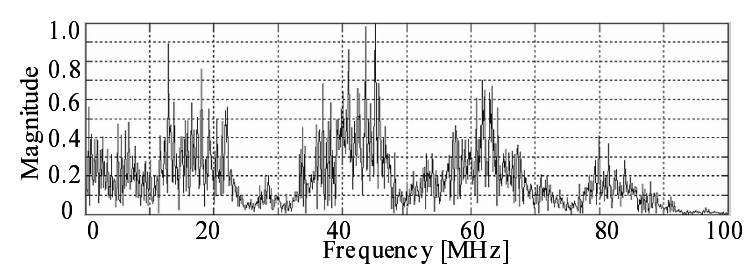

Fig. 4. Spectrum of an intra-body propagation signal.

\subsection{Conditions of measurement}

An electrode paste was used to reduce skin resistance. The distance between the electrodes for the oscillator and those for the oscilloscope was set to $10 \mathrm{~cm}$, and the distance between the signal electrode and the GND one was set to $5 \mathrm{~cm}$. Input voltage was $2.0 \mathrm{~V}_{\mathrm{P}-\mathrm{P}}$. These values were determined through preliminary experiments. Subjects were requested to sit on a chair and to keep their arm in a horizontal position. To do an efficient measurement, we utilized a wide-band colored noise signal of $0-100 \mathrm{MHz}$ generated by the oscillator as a transmission signal. An intra-body propagation signal was measured five times per day and such five spectra were averaged to suppress the fluctuation of the spectrum magnitude.

\subsection{Measurement results}

An example of the spectrum of a transmission signal is shown in Fig. 3. On the other hand, the averaged spectrum of an intra-body propagated signal is shown in Fig. 4. There are 


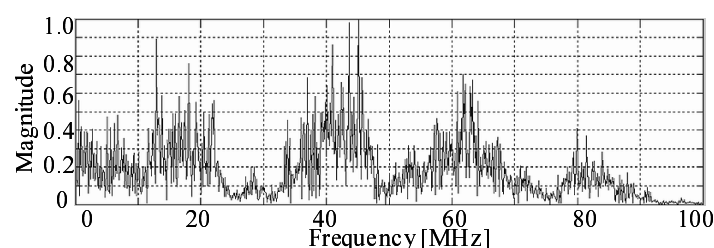

(a) The 1 st day

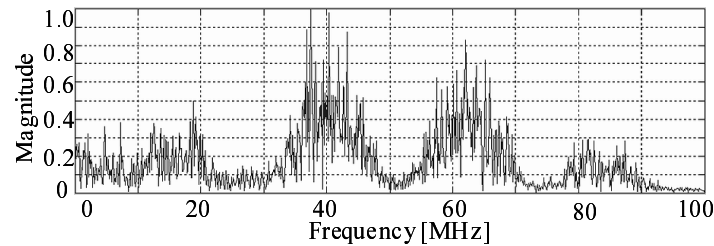

(b) The 2nd day

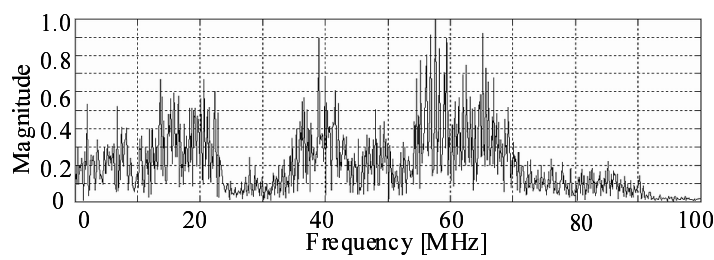

(c) The 3rd day

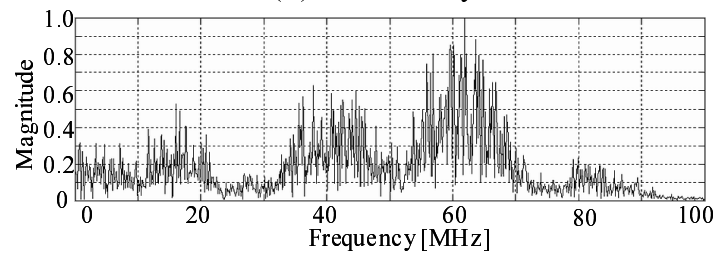

(d) The 4th day

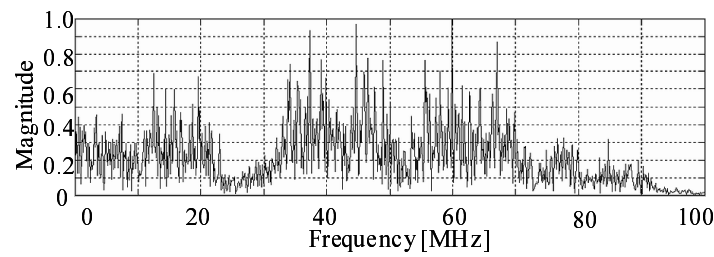

(e) The 5th day

Fig. 5. Spectra of one individual for five days.

differences between these spectra and it is certain that they were caused through the intra-body propagation. If the propagation process is different between individuals, each spectrum is different from those of others and such a difference is expected to be an individual feature in the frequency domain.

\subsection{Intra-Class Variation}

Figure 5 shows the spectra of one individual for five days. Each spectrum was obtained by averaging five spectra which were acquired during a day. Frequency elements at 40 and 60 are observed in all spectra while their variations are relatively large. These may be considered as distinctive features of a human body.

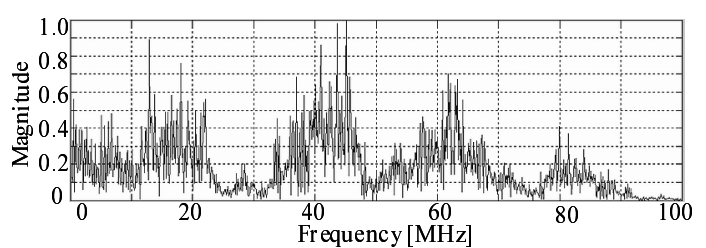

(a) Subject $A$

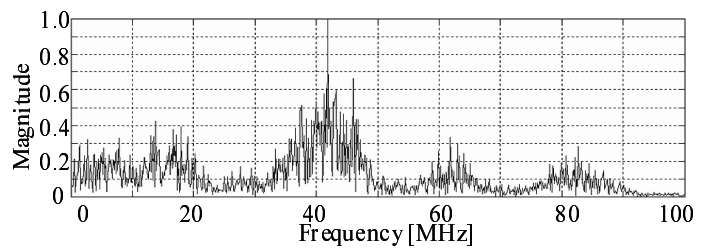

(b) Subject B

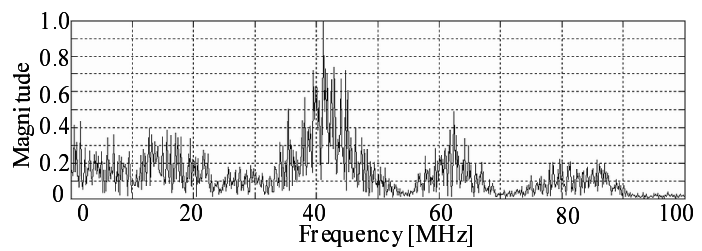

(c) Subject C

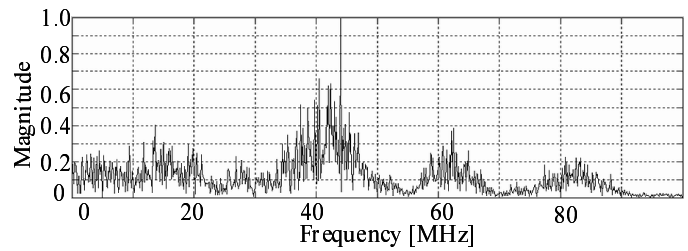

(d) Subject D

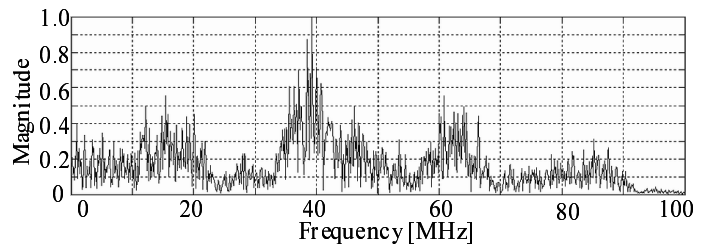

(e) Subject E

Fig. 6. Spectra of five subjects.

\subsection{Inter-Class Variation}

The same measurement was performed on five subjects; A, B, C, D, E. Their spectra at the first measurement day are shown in Fig. 6. The distribution of frequency elements resembles each other but there are some differences from one spectrum to another.

\subsection{Function of liveness detection}

In recent years, it is reported that the fingerprint counterfeited on a gummy finger was passed in some authentication system [2]. Therefore, the function of liveness detection is needed. In this subsection, we examine the spectra of intra-body propagated signals in other materials than a human body. The 


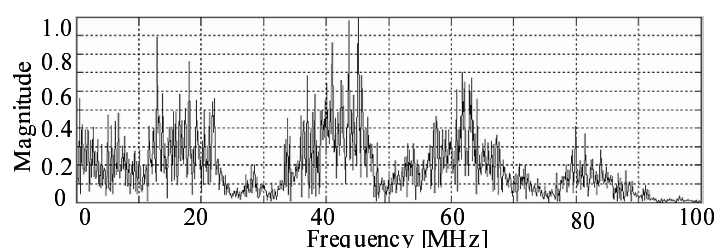

(a) Human body

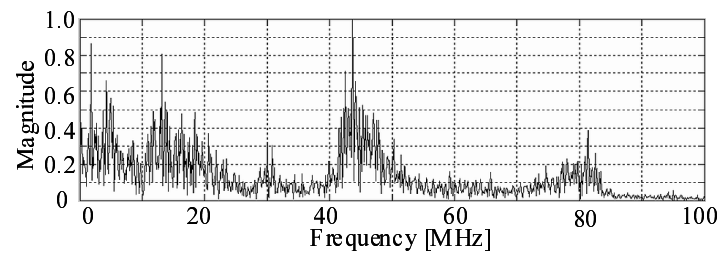

(b) Japanese radish

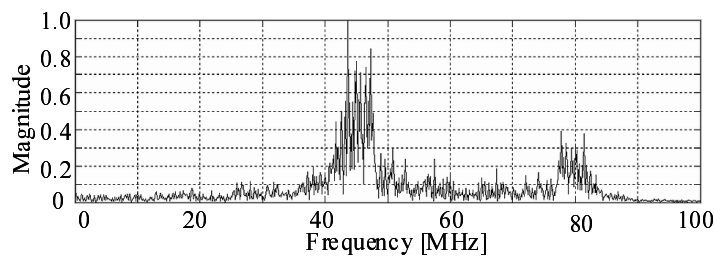

(c) Wood

Fig. 7. Spectra of intra-body propagation signals in a human body, a Japanese radish, and wood.

measurement conditions were equal to the previous ones. A Japanese radish and wood were chosen as the materials than a human body. The Japanese radish has the same amount of moisture as the human body, and its size and shape resembles a human arm. Wood was selected as an insulating material. Results are shown in Fig. 7. From these spectrum comparisons, the distribution of frequency elements was greatly different. Especially, in the case of wood, it is distinctive that frequency elements cannot be observed under $40 \mathrm{MHz}$. This suggests that liveness detection can be realized by using such a difference. Resultingly, the intra-body propagation signal makes authentication and liveness detection possible simultaneously.

\section{VERIFICATION OF SIMILARITY}

In this section, we quantitatively evaluate the degree of similarity between intra-body propagated spectra among several subjects.

\subsection{Definition of similarity}

There has been proposed a method based on the Euclidian distance for computing the degree of similarity. It is defined as $[4]$

$$
\text { Similarity }=1-\frac{\sqrt{\sum_{i=0}^{N-1}\left(a_{i}-b_{i}\right)^{2}}}{\sqrt{\sum_{i=0}^{N-1} a_{i}^{2}}+\sqrt{\sum_{i=0}^{N-1} b_{i}^{2}}}
$$

where $i$ is a frequency index (bin), $a_{i}$ is the magnitude of the frequency element of a template, $b_{i}$ is that of verification data and $N$ is the number of frequency elements (bins).

The numerator of Eq.(1) is the Euclidian distance between the template and the verification data. It is normalized by the sum of the power spectrum of the template and the verification data. If the similarity defined as Eq.(1) becomes close to 1, the verification data are considered to be similar to those of the template, that is, a genuine user.

\subsection{Evaluation based on the similarity}

The similarities between a template $\mathrm{T}$ and the verification data for four days in the subject A are summarized in Table 1, where gA1h means that the data of the subject A measured on the first day was used for verification. The template was another averaged spectrum on the first day of the subject A. The similarities of other subjects $\mathrm{B}, \mathrm{C}, \mathrm{D}$, and E comparing

Table 1. Similarities in subject A.

\begin{tabular}{c|c|c|c|c}
\hline & A1 & A2 & A3 & A4 \\
\hline $\mathrm{T}$ & 0.490 & 0.411 & 0.391 & 0.382 \\
\hline
\end{tabular}

with the template of the subject A are summarized in Table 2, where (a) is a comparison on the same measurement day and (b) is that on a different measurement day.

Table 2. Similarities in five subjects. (a) on the same measurement day

\begin{tabular}{c|c|c|c|c}
\hline & $\mathrm{B} 1$ & $\mathrm{C} 1$ & $\mathrm{D} 1$ & $\mathrm{E} 1$ \\
\hline $\mathrm{T}$ & 0.473 & 0.469 & 0.465 & 0.505 \\
\hline
\end{tabular}

(b) on a different measurement day

\begin{tabular}{c|c|c|c|c}
\hline & B2 & C2 & D2 & E2 \\
\hline $\mathrm{T}$ & 0.405 & 0.430 & 0.374 & 0.347 \\
\hline
\end{tabular}

The variation of the similarity in the subject $\mathrm{A}$, that is, the intra-class variation was $0.383-0.490$ as shown in Table 1, while the variation of the similarity among five subjects, that is, the inter-class variation was $0.347-0.430$ as shown in Table 2 (b). This result suggests the possibility of authentication using the intra- body propagation signal. 
On the other hand, the variation of the similarity among five subjects was $0.465-0.505$ as shown in Table 2 (a). In this case, the similarities in the subject $\mathrm{A}$ are less than those in others; therefore, it is hard to distinguish the subject A from others. It is necessary to examine why the intra-class variation tended to be less than the inter-class variation on the same measurement day.

\subsection{Evaluation in limited frequency ranges}

Next, we evaluate the similarity in limited frequency ranges. In this paper, we divided a frequency domain into three subbands, $0-30 \mathrm{MHz}, 30-60 \mathrm{MHz}$, and $60-100 \mathrm{MHz}$. In each subband, the similarity was calculated using Eq.(1) with limited number of frequency bins. The results are shown in Table 3 and 4.

Table 3. Similarities in subject A at each sub-band.

\begin{tabular}{c|c|c|c}
\hline & $0-30(\mathrm{MHz})$ & $30-60(\mathrm{MHz})$ & $60-100(\mathrm{MHz})$ \\
\hline A1 & 0.474 & 0.505 & 0.517 \\
\hline A2 & 0.383 & 0.434 & 0.334 \\
\hline A3 & 0.281 & 0.429 & 0.269 \\
\hline A4 & 0.342 & 0.419 & 0.320 \\
\hline
\end{tabular}

Table 4. Similarities in five subjects at each sub-band. (a) on the same measurement day

\begin{tabular}{c|c|c|c}
\hline & $0-30(\mathrm{MHz})$ & $30-60(\mathrm{MHz})$ & $60-100(\mathrm{MHz})$ \\
\hline B1 & 0.489 & 0.449 & 0.492 \\
\hline C1 & 0.478 & 0.451 & 0.485 \\
\hline D1 & 0.464 & 0.469 & 0.486 \\
\hline E1 & 0.529 & 0.470 & 0.527 \\
\hline
\end{tabular}

(b) on a different measurement day

\begin{tabular}{c|c|c|c}
\hline & $0-30(\mathrm{MHz})$ & $30-60(\mathrm{MHz})$ & $60-100(\mathrm{MHz})$ \\
\hline B2 & 0.397 & 0.418 & 0.408 \\
\hline C2 & 0.408 & 0.449 & 0.419 \\
\hline D2 & 0.343 & 0.401 & 0.313 \\
\hline E2 & 0.331 & 0.361 & 0.318 \\
\hline
\end{tabular}

Especially, in the $30-60 \mathrm{MHz}$ sub-band the variation of the similarity in the subject A was 0.419-0.505 and it was larger than that among five subjects, $0.449-0.470$ and $0.361-0.449$, regardless of when the data were acquired. Resultingly, it is expected to improve the ability of verification by comparing the spectra at the $30-60 \mathrm{MHz}$ sub-band.

\section{AUTHENTICATION EXPERIMENTS}

In this section, we evaluate the performance of the authentication using the intra-body propagation signal through authentication experiments.

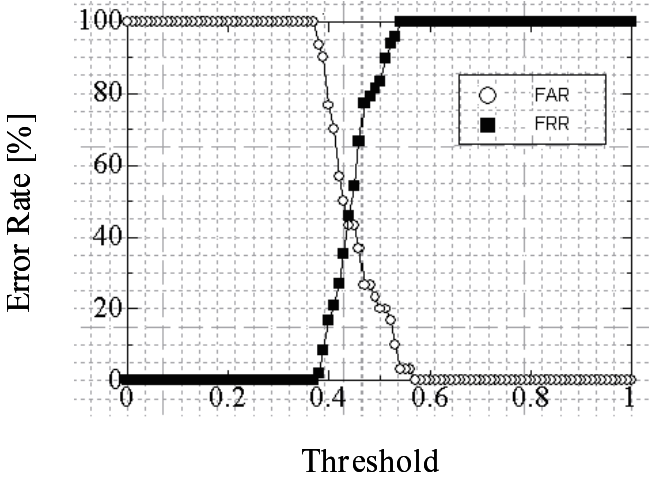

Fig. 8. Authentication results.

\subsection{Authentication results}

In the authentication, five power spectra of each individual on the first day were averaged and the result was stored as a template. The power spectrum for verification was compared with that of the template and then the similarity was larger than a threshold, the data for verification was determined as of a genuine user. Results are shown in Fig.8. The vertical and horizontal axes indicate the error rate and the threshold, respectively. In general, the performance of the authentication system is evaluated using the equal error rate (EER) at which the false acceptance rate (FAR) is equal to the false rejection rate (FRR).

From this figure, we can confirm that the EER was $44 \%$, which means the verification rate of $56 \%$.

\subsection{Authentication results in limited frequency ranges}

The authentication performance was also evaluated in limited frequency ranges, $0-30 \mathrm{MHz}, 30-60 \mathrm{MHz}$ and $60-100 \mathrm{MHz}$. Results are shown in Fig.9.

The EERs were $43 \%, 42 \%$, and $45 \%$ in the $0-30 \mathrm{MHz}, 30-$ $60 \mathrm{MHz}$, and $60-100 \mathrm{MHz}$ sub-bands, respectively. The best verification rate of $58 \%$ was obtained in the $30-60 \mathrm{MHz}$ subband. This result agrees with the evaluation result in Sect.4.3. Comparing the verification rate with that in Sect.5.1, it is confirmed that to limit the frequency range of the intra-body propagation signal is effective for evaluating the similarity.

\section{CONCLUSION}

In this paper, we proposed a new biometrics authentication using the intra-body propagation signal. It was confirmed that there were individual features in the spectrum of the intrabody propagation signal. Based on the evaluations using the similarity between spectra, we obtained the verification rate of $58 \%$ in the $30-60 \mathrm{MHz}$ sub-band. Furthermore, there were evident differences between the spectrum of a human body 
and those of other materials. This fact is expected to be effective for liveness detection.

The verification rate obtained in this paper is not high enough. More detailed examination of the spectrum, other analyses of the intra-body propagation signal, and more accurate evaluation of the similarity are needed in order to improve the verification rate.

In addition, the research of the authentication using the intra-body propagation signal has just started. At this stage, the main purpose is to evaluate the ability of the authentication using the intra-body propagation signal. Therefore, in this paper we generated and detected electromagnetic waves on a human arm with pasted electrodes as a matter of experimental convenience. In the future, we plan to examine the intra-body propagation signal on a palm without the pasted electrodes. It is assuming that we grip the knob of a door or the cellular phone by our palm. Especially, if the proposed authentication method is applied to the cellular phone, continuous (not onetime) and unconscious authentication becomes possible. While gripping such a cellular phone, the user is authenticated unconsciously. It is our future scenario.

\section{REFERENCES}

[1] A. Jain, R. Bolle, and S. Pakanti, BIOMETRICS Personal Identification in Networked Society, Kluwer Academic Publishers, Massachusetts, 1999.

[2] T. Matsumoto, H. Matsumoto, K. Yamada, and S. Hoshino, "Impact of artificial "gummy" fingers on fingerprint systems," in Proceedings of SPIE, January 2002, vol. 4677, pp. 275-289.

[3] K. Hachisuka, A. Nakata, T. Takeda, K. Siba, K. Sasaki, H. Hosaka, and Itao K, "Development of devices for communication through human bodies," Micromechatronics, Bulletin of the Horological Institute of Japan (in Japanese), vol. 46, no. 2, pp. 53-64, March 2002.

[4] R. Nagata, T. Ozaki, T. Aoki, and K. Yasuda, "A real time personal verification system based on individual characteristic extraction of hand motion," IEICE Trans. Information and Systems (in Japanese), vol. J84-D-II, no. 2, pp. 258-265, February 2001.

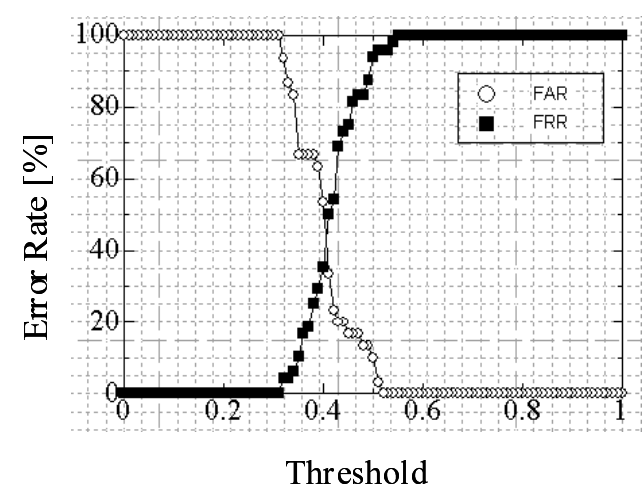

(a) $0-30 \mathrm{MHz}$

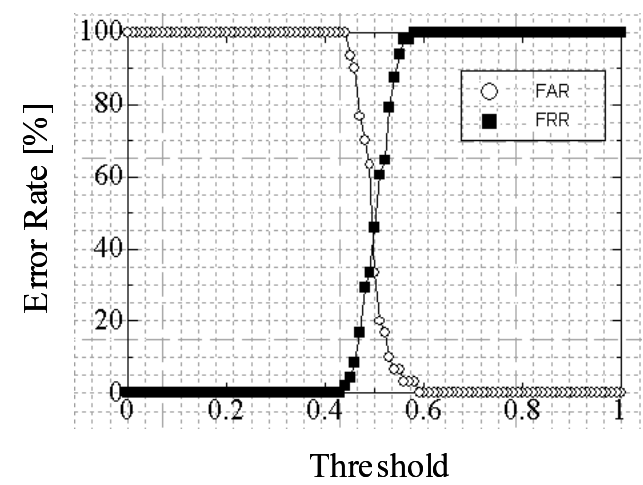

(b) $30-60 \mathrm{MHz}$

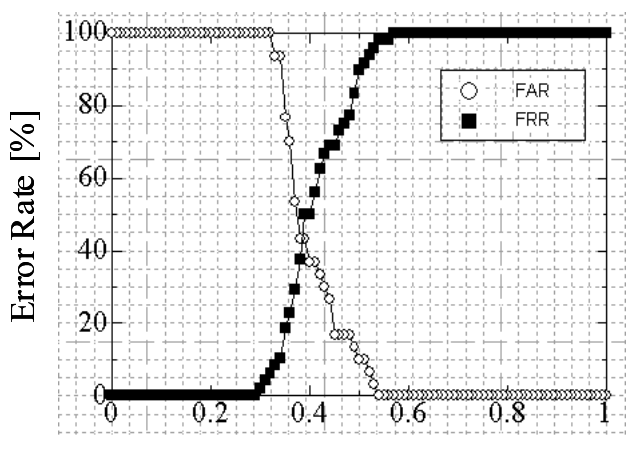

Threshold

(c) $60-100 \mathrm{Mz}$

Fig. 9. Authentication results in three sub-bands. 